\title{
UAV ONBOARD GPS IN POSITIONING DETERMINATION
}

\author{
K.N. Tahar ${ }^{\text {a, }}{ }^{*}$, S.S. Kamarudin ${ }^{\text {a }}$ \\ ${ }^{a}$ Centre of Studies for Surveying Science and Geomatics, Faculty of Architecture, Planning and Surveying, \\ Universiti Teknologi MARA, 40450 Shah Alam, Selangor Darul Ehsan, MALAYSIA - \\ khairul0127@salam.uitm.edu.my, sarah_kamarudin@hotmail.com
}

\section{Commission I, ICWG I/Vb}

KEY WORDS: Onboard GPS, Observation, Error, Analysis

\begin{abstract}
:
The establishment of ground control points is a critical issue in mapping field, especially for large scale mapping. The fast and rapid technique for ground control point's establishment is very important for small budget projects. UAV onboard GPS has the ability to determine the point positioning. The objective of this research is to assess the accuracy of unmanned aerial vehicle onboard global positioning system in positioning determination. Therefore, this research used UAV onboard GPS as an alternative to determine the point positioning at the selected area. UAV is one of the powerful tools for data acquisition and it is used in many applications all over the world. This research concentrates on the error contributed from the UAV onboard GPS during observation. There are several points that have been used to study the pattern of positioning error. All errors were analyzed in world geodetic system 84coordinate system, which is the basic coordinate system used by the global positioning system. Based on this research, the result of UAV onboard GPS positioning could be used in ground control point establishment with the specific error. In conclusion, accurate GCP establishment could be achieved using UAV onboard GPS by applying a specific correction based on this research.
\end{abstract}

\section{INTRODUCTION}

Drones or UAVs are very popular among civilian, military and educational researchers. Nowadays, most surveillance applications use UAV in order to reduce risk during the operation. The surveillance application has been used by police, fire, environmental, coastal guard departments. The cost of surveillance can also be reduced with UAV drones technology. UAV has also been applied in many surveying and mapping fields such as photogrammetric survey, engineering survey, hydrographic survey and cadastral survey. The accuracy of mapping is always based on the specific applications.

UAV Onboard GPS has been investigated by many researchers over the world (Daakir, Pierrot-Deseilligny, Bosser, Pichard, \& Thom, 2015). Basically, UAV onboard GPS could give the approximate coordinates during a flight mission. It is very important to estimate the position and attitude of UAV, especially during an autonomous mission (Turner, Lucieer, \& Wallace, 2014). It is because the pilot could handle any circumstances during emergency cases such as signal loss or bad weather. The UAV onboard GPS has the same operation with the common GPS, which relies on global navigation satellite systems (Rehak, Mabillard, \& Skaloud, 2013). There are many types of UAV onboard GPS available in the market and each type has its own strength and capabilities to acquire signal from the satellite. Basically, the satellite constellation during flight mission is very important in order to give accurate coordinates. Therefore, the user needs to study the satellite constellation before flight mission. There are many studies that have been conducted to overcome the bad satellite reception and signal loss during a flight mission. One of the methods is using fail safe mode where the UAV will return to launch when a signal is lost from the transmitters, GPS bad reception or low battery. This function can prevent bad things from happening to the UAV and it can land safely. There are many designs for UAV onboard GPS which are available in the market (Figure 1). Each design has its specific strength to receive signal from different satellites. The available satellite signals are such as GPS, GLONASS, Galileo, BeiDou, QZSS and SBAS.

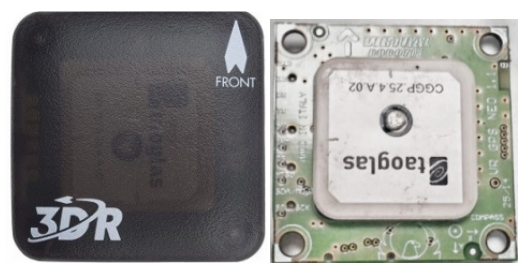

Figure 1. UAV onboard GPS, 3DR VRX GPS NEO-M8

Four considerations need to be taken into account when it comes to the onboard GPS positioning, namely compliance, accuracy, interference and errors (Gupta, 2015). Recently, many new regulations are considered in relation to UAV performance and its freedom, especially in sensitive areas such as military base, airport and civilian areas. UAV operators need to follow the regulation and avoid the sensitive areas during flight planning. The accuracy of UAV always depends on the specific application. Some UAVs are designed for hobby purposes, which do not need precise positioning during flight while military UAVs require high accuracy of positioning in order to complete the mission (Tahar, 2015). The accuracy of UAV depends on the signal received from the satellite platform. The cheap GPS only can receive weak signal and selected signal only whereas the expensive GPS can usually support and receive many types of signal, which are able to do the positioning correction in real time. The application, such as outdoor medical drones, requires accurate positioning to deliver the medical equipment to the patient's location (Morris, 2014). Inaccurate position along the designed waypoints could cause the death of the patient (Shahbaz, 2015). Therefore, GPS reception is very important in some cases which require high accuracy positioning. Signal interference during flight mission is a very annoying circumstance especially for a small budget project. The signal interference might be caused by the transmission lines, power system and telecommunication tower. The transmitter frequency is very sensitive to other signals and 
it might cause signal loss during flight. The UAV operator needs to avoid the potential interference at the flight area. The UAV can be affected by many errors which come from different factors such as wind, solar weather, electromagnetic waves and the surrounding environment. All of these considerations could influence the accuracy of UAV positioning. Therefore, more research needs to be carried out in order to determine the best practice to acquire high UAV positioning.

This research focuses on the UAV positioning for ground control point establishment. The objective of this research is to assess the accuracy of unmanned aerial vehicle onboard global positioning system in positioning determination.

\section{MATERIAL AND METHODS}

This research is conducted at Universiti Teknologi MARA area at latitude 3.067812 degree and longitude 101.500931 degree. This university is located about 30 kilometres from Kuala Lumpur, the capital city of Malaysia or a 40-minute drive. The area of this university is illustrated in Figure 2.

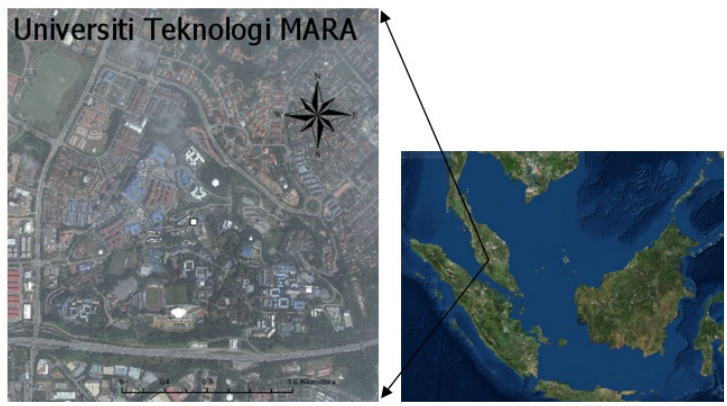

Figure 2. Research Area, Universiti Teknologi MARA

This research involves four main phases, namely preparation, observation, processing and analysis. The preparation includes the UAV installation and setup, where the DJI F450 framework model with some modifications is used for this research. This model is complete with flight control, battery, power module, GPS receiver, electronic speed controller, four motors and transmitter receiver. The modified DJI F450 model is shown in Figure 3.

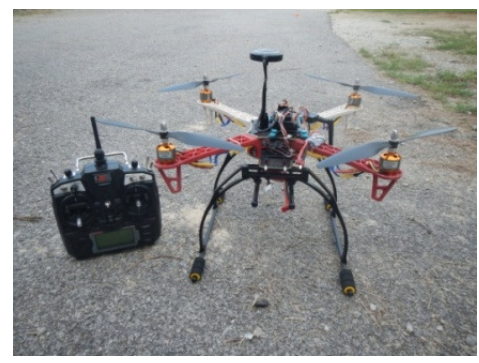

Figure 3. UAV

This model is a low cost system which can be afforded by most of people. This kind of model can be classified in microcategory because it is below five kilograms. The preparation also includes the selection and establishment of ground control points at the selected area. 36 points have been identified and used for this research. All points were located at the open area and free from multipath distraction. The GPS planning is also considered at this phase where the GPS constellation for each observation is configured from almost the same satellites in order to maintain the accuracy of the observation points. The time of observation is based on the estimated time of the same satellite for every single day. The second phase is about UAV onboard GPS observation at the selected points. The observation took about 30 minutes and one hour observation time for each point. This technique is almost similar to the existing technique in GPS observation where it is known as rapid static and static methods. The reason this interval is chosen is to see the pattern of error at each point, whether it has the same behaviour or random pattern. The third phase discusses data processing where all UAV onboard GPS observation data were processed to determine the blunders or outliers data in the observation data. The conversion from raw file to the text file was also done in this phase. Finally, all the observation data were analysed to assess the accuracy of UAV onboard GPS at the selected points. The behaviour and pattern of error were also studied for future references. The overall methodology for this research is illustrated in Figure 4.

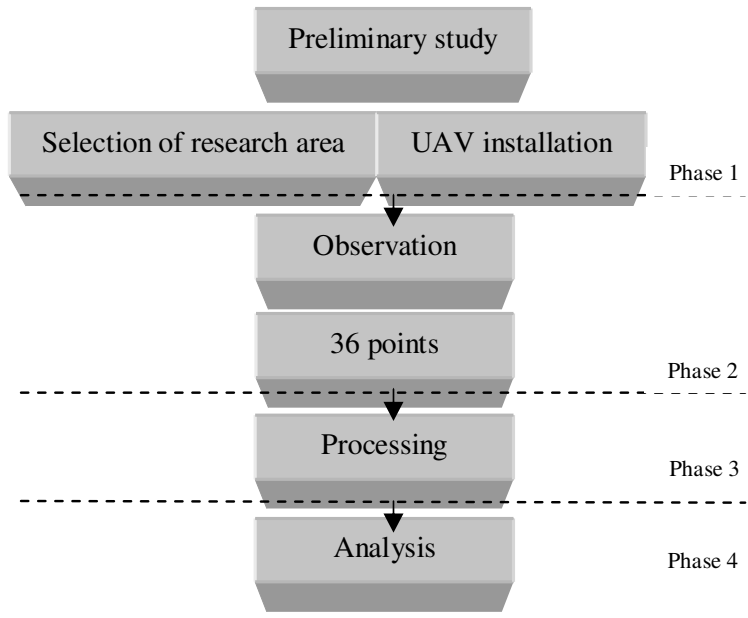

Figure 4. Research Methodology

This observation is only based on single differencing between satellites. In theory, the pseudorange, carrier phase and Doppler frequency can be affected by satellite and receiver clocks error, atmospheric effects and orbital error (Ebadi, 2000). Single differencing between receivers or satellite could eliminate these errors. The basic equation for differencing between satellites involves twelve parameters, including speed of light (c), orbital error $(\mathrm{d} \rho)$, satellite clock error $(\mathrm{dt})$, carrier wavelength $(\lambda)$, ionospheric error $\left(\mathrm{d}_{\text {ion }}\right)$, troposheric error $\left(\mathrm{d}_{\text {trop }}\right)$, carrier phase measurement noise and multipath $(\varepsilon \Phi)$, unknown integer cycle ambiguity $(\mathrm{N})$, differences between pseudorange and carrier phase observation $(\lambda N)$, observed carrier phase $(\Phi)$, unknown satellite receiver range $(\rho)$ and code measurement noise and multipath $(\varepsilon \rho)$. The concept of single differencing between satellites is illustrated in Figure 5. The single differencing between satellites can eliminate receiver clock error.

$\nabla \rho=\nabla \rho+\nabla$ d $\rho+$ c $\nabla$ dt $+\nabla$ dion $+\nabla$ dtrop $+\nabla \varepsilon p$

$\nabla \Phi=\nabla \rho+\nabla d \rho+c \nabla d t+\lambda \nabla N-\nabla$ dion $+\nabla d$ trop $+\nabla \varepsilon \Phi \quad$ eq. 2

$\nabla \Phi=\nabla \rho+\nabla$ d $\rho+$ c $\nabla$ dt $-\nabla$ dion $+\nabla$ dtrop $+\nabla \varepsilon \Phi$

where

$\nabla=$ denotes a single difference operator between satellites 


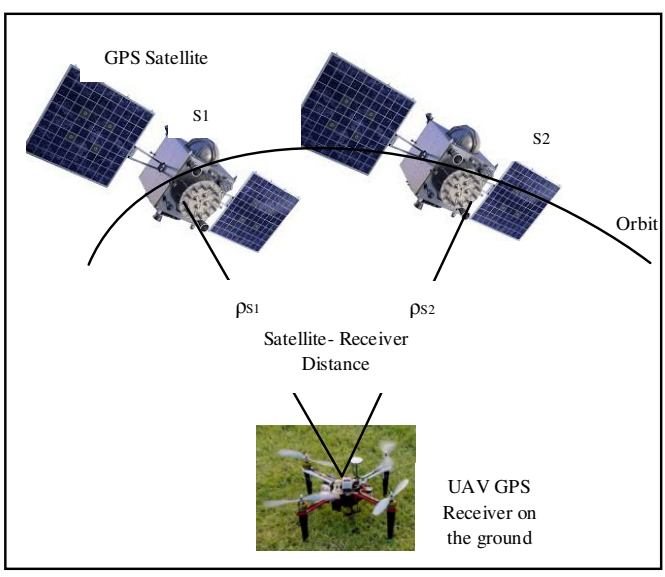

Figure 5. Differencing between satellites

\section{RESULTS AND ANALYSIS}

This research only concentrates on the accuracy of point positioning at the selected research area. The observation was solely conducted based on UAV onboard GPS for low cost UAV system. As mentioned before, there are about 36 points that have been randomly distributed at the selected research area and each point was observed at 30 minutes and one hour of observation. The results were divided into three categories, which consist of latitude, longitude and height. The latitude and longitude were measured in degree unit measurement while the height in meter. The height refers to the ellipsoid surface with respect to world geodetic system. The mean for each point of observation data were calculated in order to determine the behaviour of onboard GPS data at the specific location. Figure 6 shows the mean for latitude, longitude and height for each point observation, which is based on one-hour observation. It was found that the range of mean for latitude is about \pm 0.16 second, longitude is about \pm 0.18 second and height is about \pm 13.072 meters. Based on these results, the UAV onboard has almost similar results in terms of latitude and longitude while the height shows the big range. This might be due to the capability of UAV onboard GPS component to calculate the geometric position in vertical perspective.

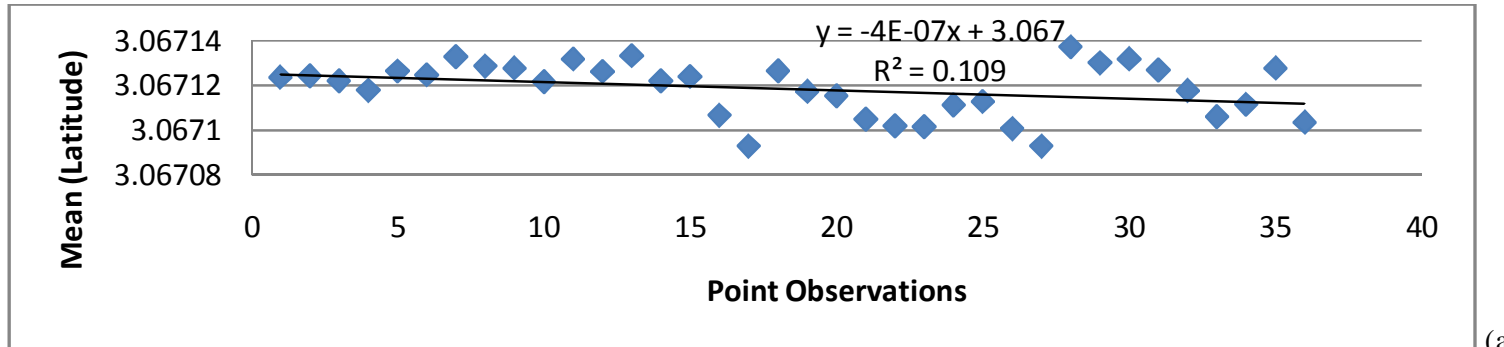

(a)
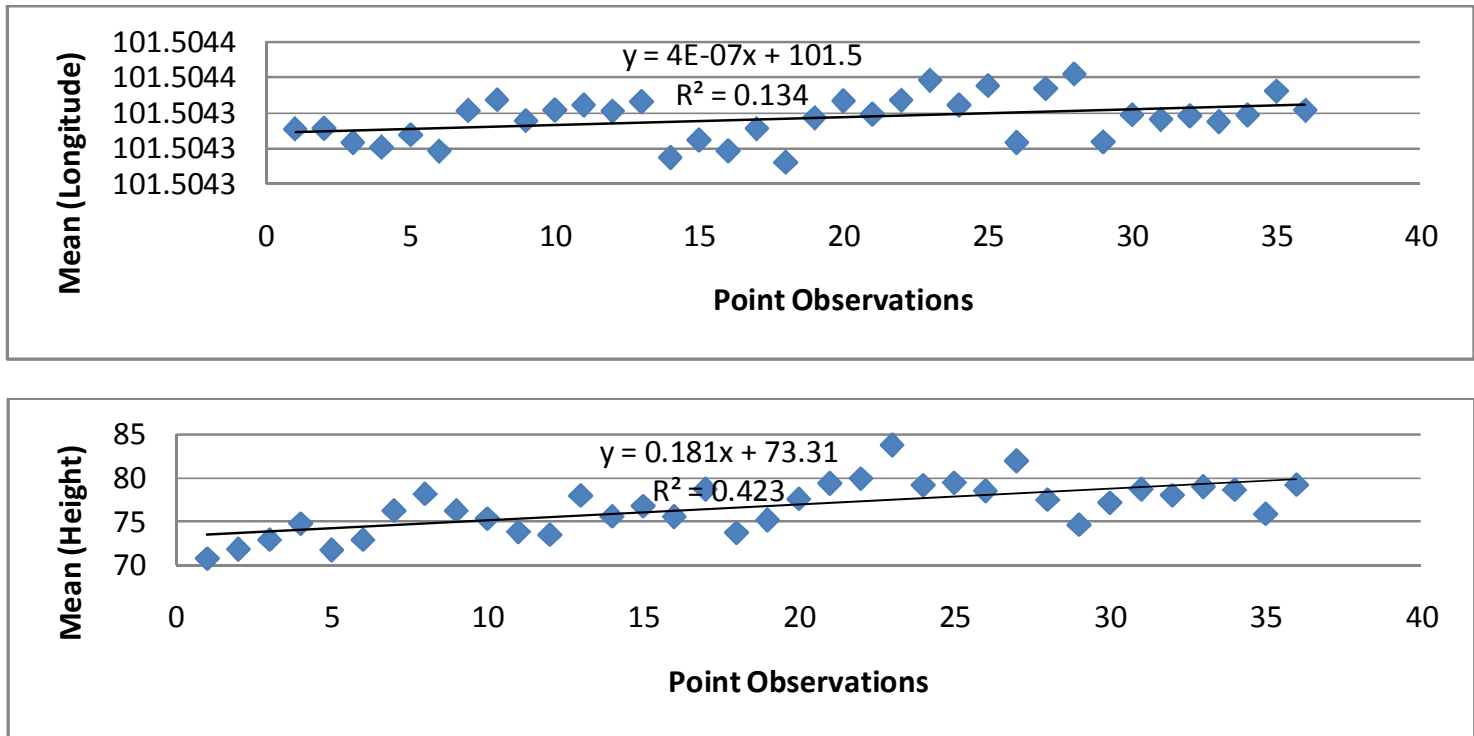

(c)

Figure 6. Mean for latitude, longitude and height (one hour)

Figure 7 illustrates the mean result for latitude, longitude and height for 30 minutes observation. The result shows that the range of mean for latitude is about \pm 0.17 second, longitude is about \pm 0.2 second and height is about \pm 13.647 meters. Based on the one hour and 30 minutes of observation results, the difference for latitude is only about \pm 0.01 second, longitude is about \pm 0.02 second and height is about \pm 0.575 meters.
Therefore, as the main finding of this research, the time of observation for one hour and 30 minutes does not influence the accuracy of the positioning in this research. The UAV onboard GPS data need an external correction data in order to obtain the correct and accurate position. The next analysis for this research is derived from residual mean square error (Figure 8 and Figure 9). 

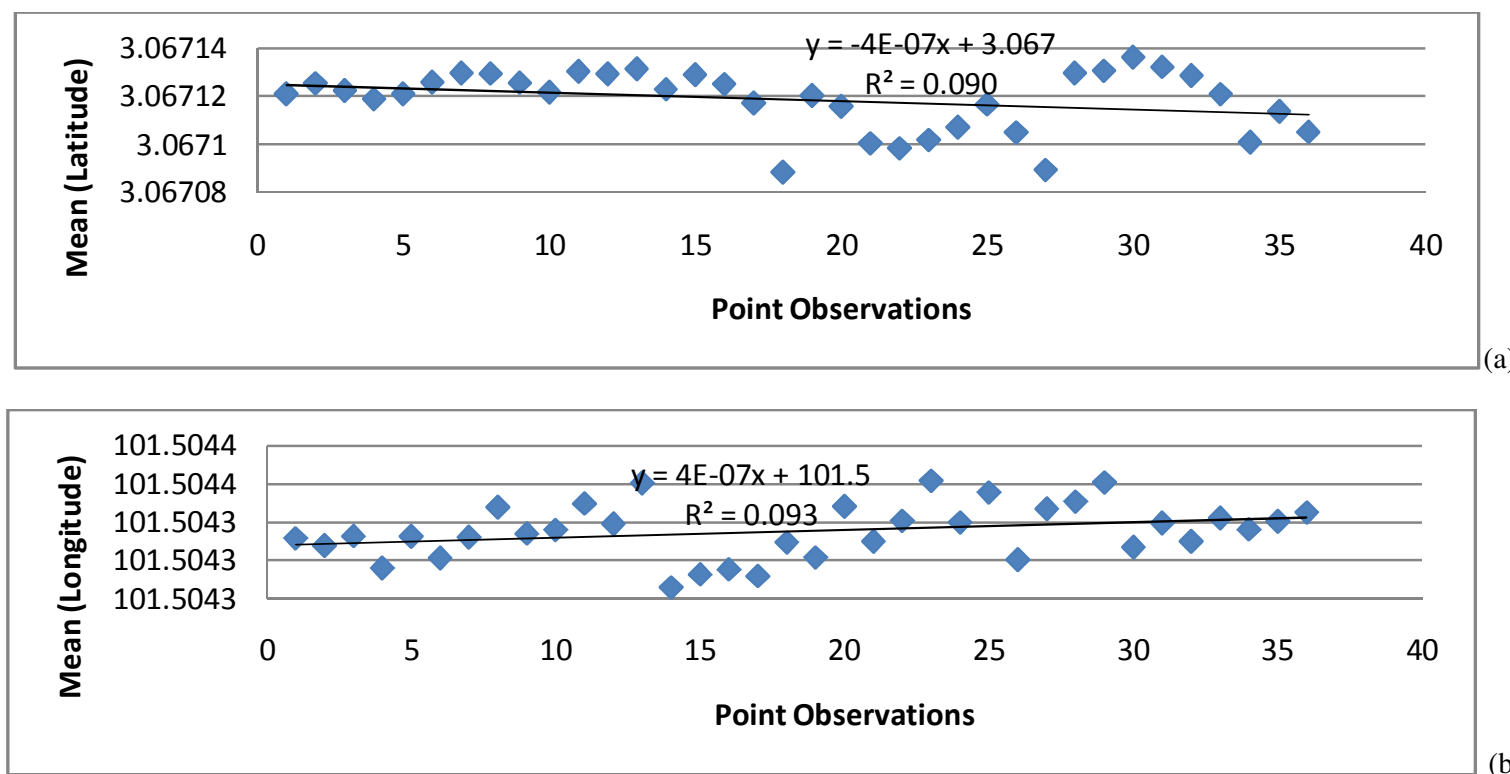

(b)

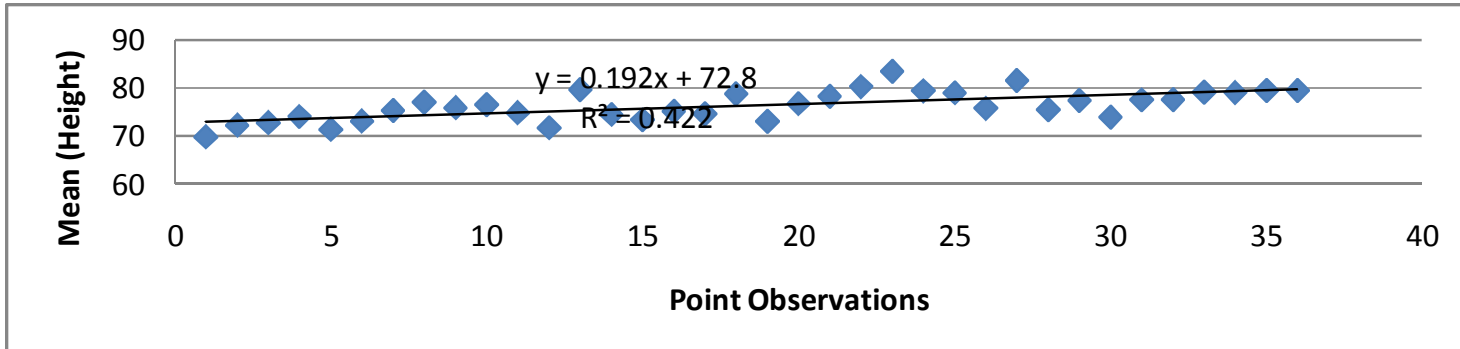

Figure 7. Mean for latitude, longitude and height (30 minutes)

(c)

Figure 8 describes the residual mean square error (RMSE) result for latitude, longitude and height for one hour observation. The RMSE for latitude shows 13 points observation exceed 0.04 second while the rest maintain under the 0.04 second mark. The RMSE for longitude describes 20 points observation exceed 0.04 second and 16 points observation remain under 0.04 second. The RMSE for height illustrates 21 points observation above 15 meters and 15 points below 15 meters. The result for latitude and longitude shows inconsistent pattern where there is a huge difference between peak and low value, which is about \pm 0.11 second and \pm 0.08 second for latitude and latitude respectively. Meanwhile, the height shows irregular pattern but the peak and low does not reflect the contrasting pattern. It might be due to the temporary loss of the satellite signal during observation where and UAV onboard GPS was not able to acquire the signal for a certain duration.

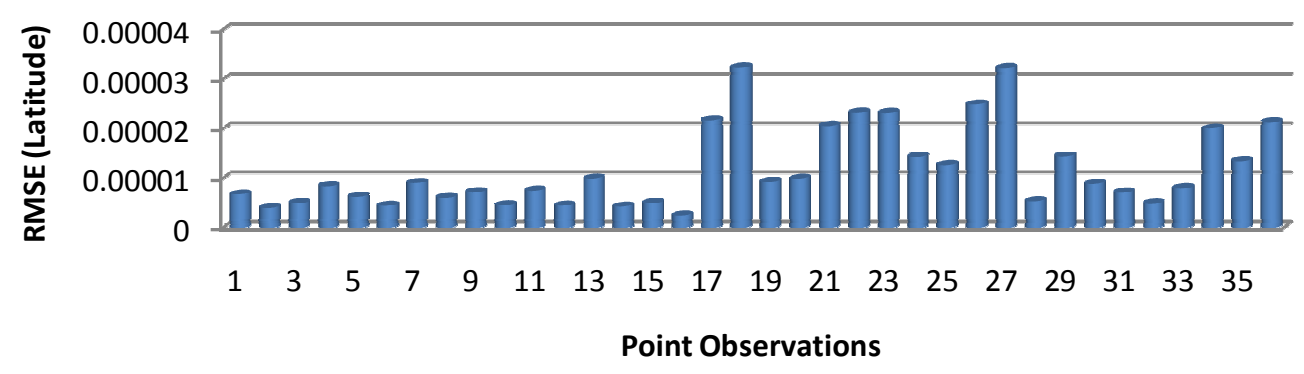




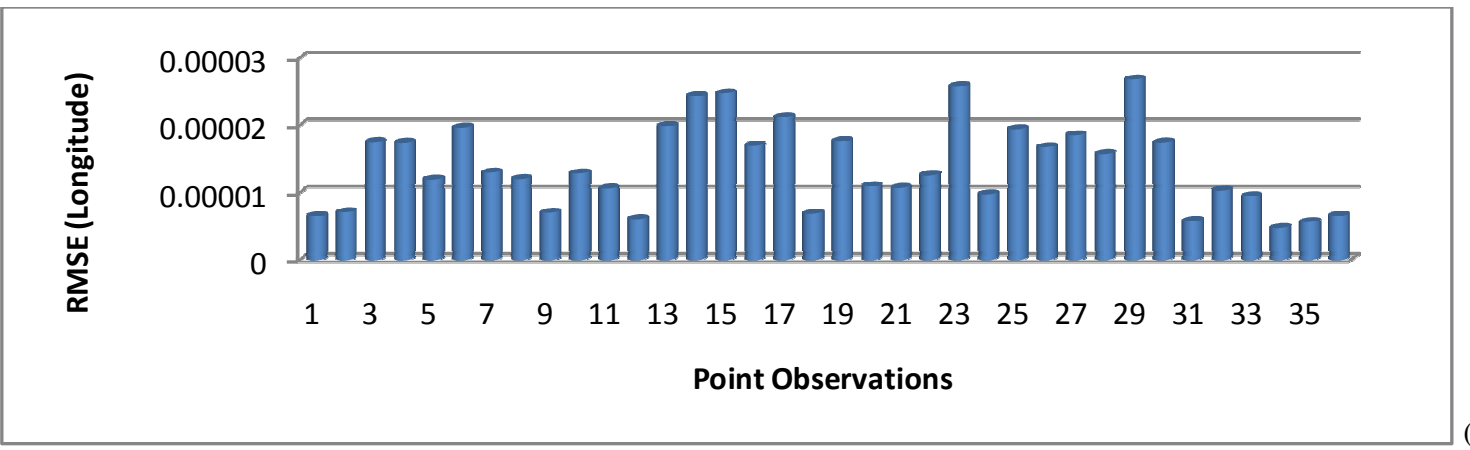

(b)

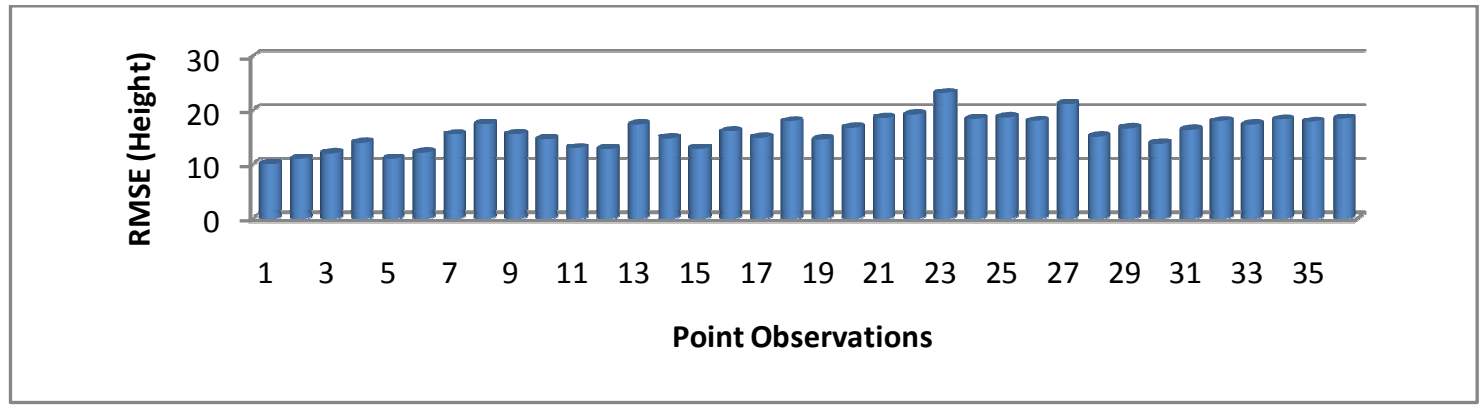

(c)

Figure 8. Residual Mean Square Error for latitude, longitude and height (one hour)

Figure 9 describes the RMSE for latitude, longitude and height results. There are about 11 points that exceed 0.04 second for latitude, 18 points that exceed 0.04 second for longitude and 18 points above 15 meters for height. Similar to one-hour observation, 30-minute observation result also illustrates the inconsistent graph pattern for latitude and longitude. The difference in peak and low value is about +0.12 second and \pm 0.1 second for latitude and longitude respectively. The height records an irregular pattern where the range between peak and low value is about +13.734 meters
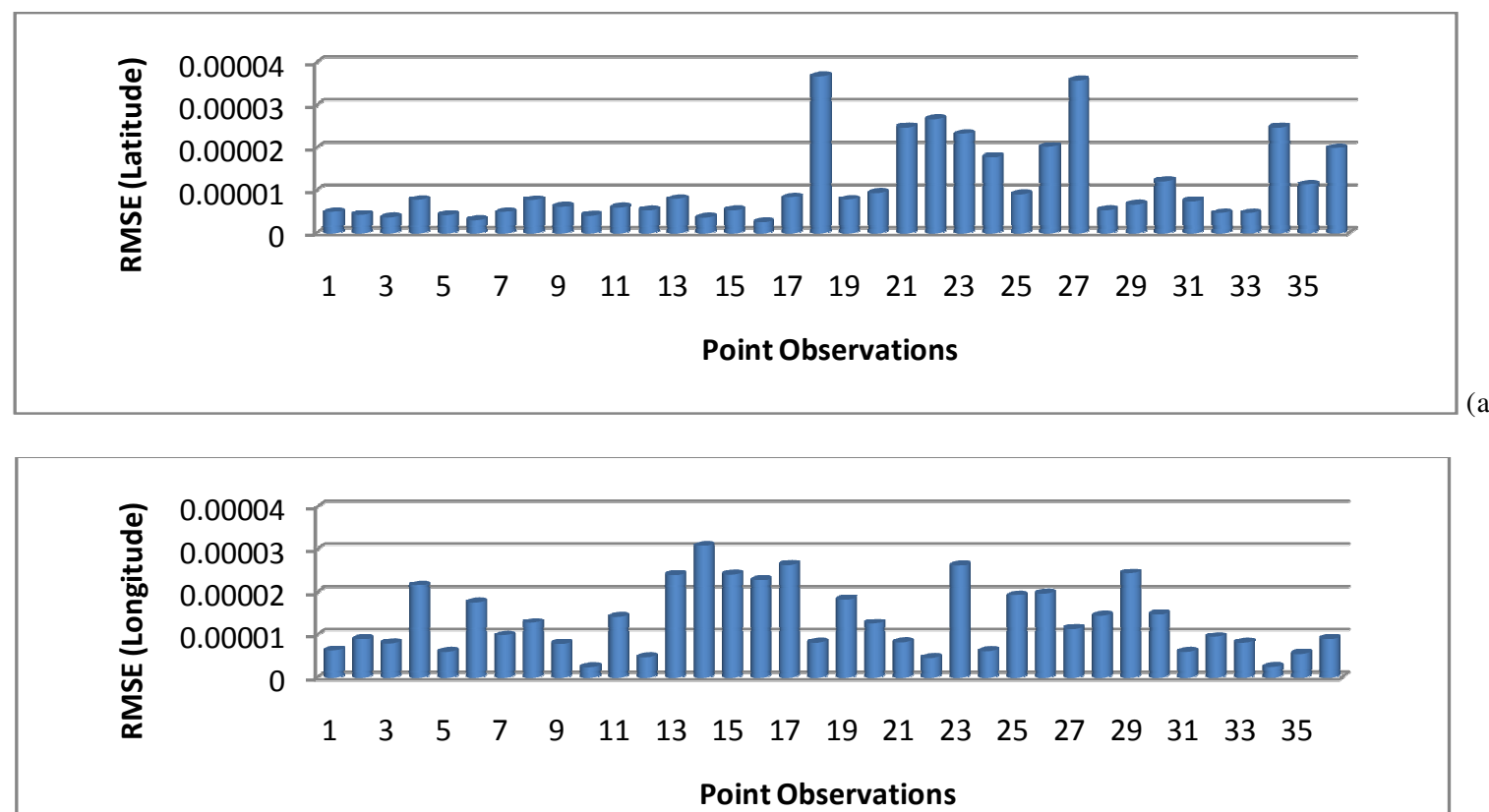

(b) 


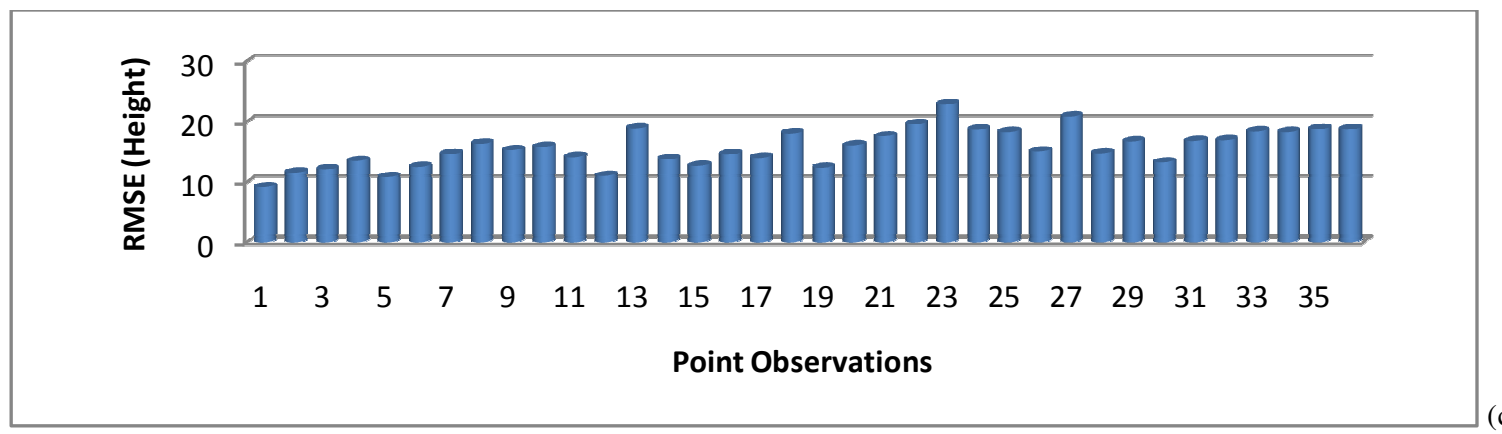

Figure 9. Residual Mean Square Error for latitude, longitude and height (30 minutes)

\section{CONCLUSIONS AND RECOMMENDATIONS}

This research has investigated the capability of UAV onboard GPS in positioning determination. There are two observation times that have been considered in this research, i.e. one hour and 30 minutes. The observation data were analysed using mean and RMSE method. It shows that the mean results describe the difference between latitude and longitude and height for one-hour and 30-minute observation is about 0.01 second, 0.02 second and 0.575 meters respectively. The RMSE results between latitude, longitude and height for one-hour and 30-minute observation are about 0.01 second, 0.02 second and 0.576 meters respectively. It can be concluded that, the accuracy of UAV onboard GPS has invariable results for this research. The data correction from the virtual reference station or real time kinematic data may increase the accuracy results. In future work, the investigation on the accuracy of UAV onboard GPS using data correction from virtual reference station and real time kinematic data will be conducted.

\section{ACKNOWLEDGEMENTS}

Faculty of Architecture, Planning and Surveying Universiti Teknologi MARA (UiTM), Research Management Institute (RMi) and Ministry of Higher Education (MOHE) are greatly acknowledged for providing the fund RAGS 600-RMI/RAGS 5/3 (241/2014), RAGS/1/2014/TK09/UITM/3 to enable this research to be carried out. The authors would also like to thank the people who were directly or indirectly involved in this research.

\section{REFERENCES}

Daakir, M., Pierrot-Deseilligny, M., Bosser, P., Pichard, F., \& Thom, C., 2015. UAV Onboard Photogrammetry and GPS Positioning for Earthworks. The International Archives of the
Photogrammetry, Remote Sensing and Spatial Information Sciences, Volume XL-3/W3, 2015 ISPRS Geospatial Week 2015, pp. 293-298. La Grande Motte, France: ISPRS.

Ebadi, H., 2000. Positioning with GPS. K.N.Toosi University of Technology.

Gupta, R., 2015. Four key GPS Test Consideration for Drone and UAV Developers. Spirent.

Morris, L., 2014. In a heartbeat: Ambulance Drone designed to cut cardiac arrest response times. Electronic Engineering Journal .

Rehak, M., Mabillard, R., \& Skaloud, J., 2013. A Micro-UAV with the Capability of Direct Georeferencing. International Archives of the Photogrammetry, Remote Sensing and Spatial Sciences $X L-1 / W 2$, pp. 317-323. Rostock, Germany: ISPRS.

Shahbaz, S., 2015. Ambulance Drone Support System (ADSS). International Journal of Engineering Science and Innovative Technology, pp. 80-89.

Tahar, K.N., 2015. Multi Rotor UAV at Different Altitudes for Slope Mapping Studies. The International Archives of the Photogrammetry, Remote Sensing and Spatial Information Sciences, Volume XL-1/W4, pp. 9-16. Toronto, Canada: ISPRS.

Turner, D., Lucieer, A., \& Wallace, L., 2014. Direct Georeferencing of Ultrahigh-Resolution UAV Imagery. Geoscience and Remote Sensing, IEEE Transactions on 52(5), pp. 2738-2745. 